\title{
Water Masers Toward Star-Forming Regions in the Bolocam Galactic Plane Survey
}

\author{
Miranda K. Dunham ${ }^{1}$ and The BGPS Team \\ ${ }^{1}$ Department of Astronomy, Yale University, \\ P. O. Box 208101, New Haven, CT, USA \\ email: miranda.dunham@yale.edu
}

\begin{abstract}
We present preliminary results of a search for $22 \mathrm{GHz}$ water masers toward 1400 star-forming regions seen in the Bolocam Galactic Plane Survey (BGPS) using the Green Bank Telescope (GBT). The BGPS is a blind survey of the Northern Galactic plane in $1.1 \mathrm{~mm}$ thermal dust emission that has cataloged star-forming regions at all evolutionary stages. Further information is required to determine the stage of each BGPS source. Since water masers are produced by outflows from low and high-mass star forming regions, their presence is a key component of determining whether the BGPS sources are forming stars and which evolutionary stage they are in. We present preliminary detection statistics, basic properties of the water masers, and correlations with physical properties determined from the $1.1 \mathrm{~mm}$ emission and ammonia observations obtained concurrently with the water masers on the GBT.
\end{abstract}

Keywords. ISM: molecules, masers, radio lines: ISM, stars: formation, surveys

One of the main outstanding problems of high-mass star formation is the lack of an evolutionary sequence similar to that of low-mass star formation (e.g. Shu, Adams \& Lizano 1987). Several evolutionary sequences have been proposed recently (e.g. Battersby et al. 2011; Chambers et al. 2009) that are based on the presence or absence of 8 and $24 \mu \mathrm{m}$ emission, $\mathrm{H}_{2} \mathrm{O}$ and $\mathrm{CH}_{3} \mathrm{OH}$ masers, $4.5 \mu \mathrm{m}$ outflows, ultra-compact HII regions, as well as a determination of the dust temperature. A detailed study of the physical properties of a large sample of star-forming regions with different combinations of these evolutionary indicators is necessary to solidify an evolutionary sequence. A large scale, unbiased catalog of star-forming regions is of paramount importance in such a study.

The BGPS is a blind survey of the northern Galactic plane in optically thin $1.1 \mathrm{~mm}$ thermal dust continuum. The BGPS has detected 8,358 sources (Rosolowsky et al. 2010) and follow-up observations in dense gas tracers return high detection rates $(72 \%$ in NH3, Dunham et al. 2010, 2011; 77\% in HCO+, Schlingman et al. 2011) demonstrating that the high column density features in the BGPS correspond to high volume density regions. The Bolocam Galactic Plane Survey (BGPS) provides an ideal catalog of high-mass starforming regions to solidify an evolutionary sequence.
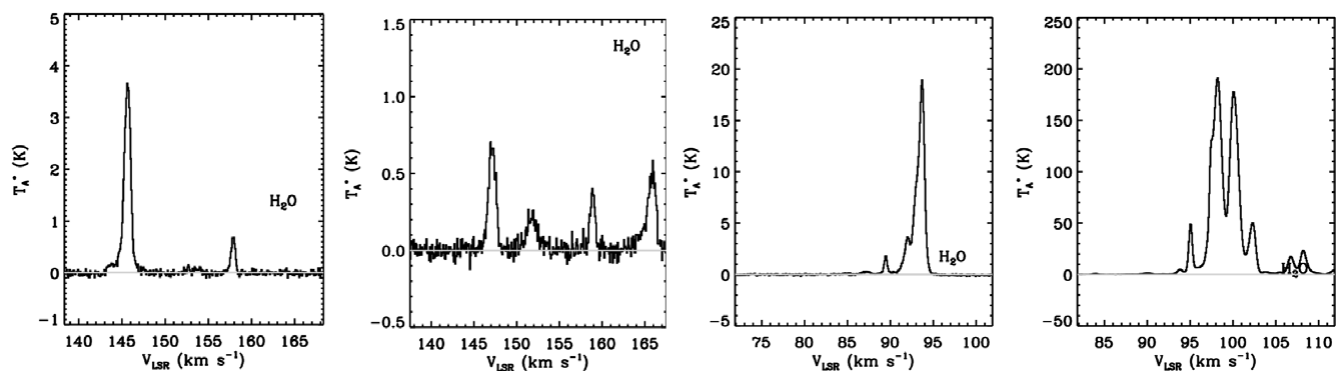

Figure 1. $\mathrm{H}_{2} \mathrm{O}$ spectra for four BGPS sources, from left to right 1311, 1316, 4760, and 4764 . 

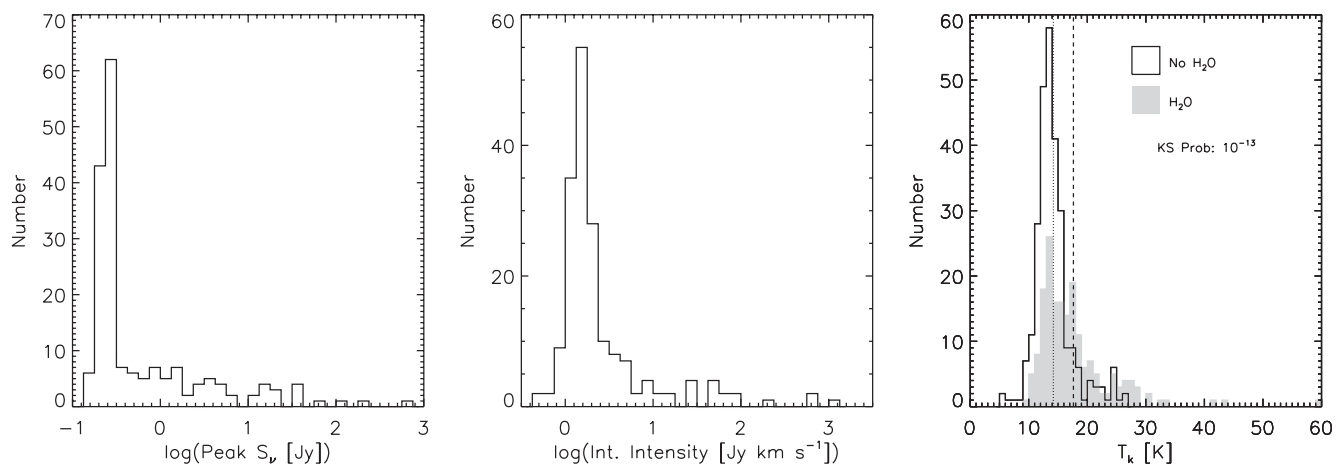

Figure 2. Distrbution of the peak flux densities (left) and integrated intensity (middle) of the $\mathrm{H}_{2} \mathrm{O}$ masers observed toward the BGPS sources. Right: Distribution of gas kinetic temperatures as measured from $\mathrm{NH}_{3}$ of BGPS sources with (gray filled) and without (solid line) $\mathrm{H}_{2} \mathrm{O}$ maser emission (gray filled). The dashed and dotted lines denote the mean temperatures of BGPS sources with and without $\mathrm{H}_{2} \mathrm{O}$, respectively.

We have used the Green Bank Telescope (GBT) to observe single pointings of $\mathrm{NH}_{3}(1,1)$, $(2,2),(3,3)$ and the $22 \mathrm{GHz} \mathrm{H}_{2} \mathrm{O}$ line toward 1400 BGPS sources. The $\mathrm{NH}_{3}$ observations provide a determination of the gas temperature and the $\mathrm{H}_{2} \mathrm{O}$ observations address the presence/absence of $\mathrm{H}_{2} \mathrm{O}$ masers within the $30^{\prime \prime}$ GBT beam. Here we present preliminary $\mathrm{H}_{2} \mathrm{O}$ results from a subset of 456 BGPS sources.

Of the 456 BGPS sources considered, 182 (40\%) exhibit $\mathrm{H}_{2} \mathrm{O}$ emission above a $5 \sigma$ integrated intensity and a $4 \sigma$ peak flux density threshold. Figure 1 shows example $\mathrm{H}_{2} \mathrm{O}$ spectra from four BGPS sources spanning a range of maser strength and number of components. Figure 2 shows distributions of various observed properties of the $\mathrm{H}_{2} \mathrm{O}$ masers, as well as the distributions of gas kinetic temperatures for BGPS sources with and without $\mathrm{H}_{2} \mathrm{O}$ maser emission. Sources with $\mathrm{H}_{2} \mathrm{O}$ masers have a warmer mean kinetic temperature $(17.6 \mathrm{~K})$ compared to sources without $\mathrm{H}_{2} \mathrm{O}$ masers $(14.2 \mathrm{~K})$, suggesting that sources with $\mathrm{H}_{2} \mathrm{O}$ masers are more evolved since kinetic temperature increases along the proposed evolutionary sequences.

In the future, we will expand this analysis to include all 1400 BGPS sources with GBT observations. With the large sample size provided by the BGPS, we will explore trends in physical properties as a function of presence/absence of $\mathrm{H}_{2} \mathrm{O}$ masers. Beyond this study of $\mathrm{H}_{2} \mathrm{O}$ masers, we will compare the BGPS sources with catalogs of the remaining evolutionary indicators in order to solidify an evolutionary sequence for high-mass star formation.

\section{References}

Aguirre, J. E., Ginsburg, A. G., Dunham, M. K., et al. 2011, ApJS, 192, 4

Battersby, C., Bally, J., Ginsburg, A., et al. 2011, A\&A, 535, A128

Chambers, E. T., Jackson, J. M., Rathborne, J. M., \& Simon, R. 2009, ApJS, 181, 360

Dunham, M. K., Rosolowsky, E., Evans, N. J., II, et al. 2010, ApJ, 717, 1157

Dunham, M. K., Rosolowsky, E., Evans, N. J., II, Cyganowski, C., \& Urquhart, J. S. 2011, ApJ, 741,110

Rosolowsky, E., Dunham, M. K., Ginsburg, A., et al. 2010, ApJS, 188, 123

Schlingman, W. M., Shirley, Y. L., Schenk, D. E., Rosolowsky, E., Bally, J., et al. 2011, ApJS, 195, 14

Shu, F. H., Adams, F. C., \& Lizano, S. 1987, ARAA, 25, 23 